\title{
Reversible Susceptibility of Hematite Carrying Various Types of Remanent Magnetization and Memory
}

\author{
Kazuo Kobayashi and R. W. Smith \\ Department of Earth and Planetary Sciences, University of Pittsburgh, Pittsburgh, U.S.A.
}

\begin{abstract}
The reversible susceptibility of large single crystals of hematite carrying different intensities of various types of remanent magnetization, i.e., IRM, TRM and ARM has been measured in a small A C magnetic field $(\mathrm{H} \doteqdot 3 œ)$ using a transformer bridge. In a stoichiometric $\alpha \mathrm{Fe}_{2} \mathrm{O}_{3}$ specimen, reversible susceptibility has also been measured before and after cooling and heating through the Morin transition. The results of these experiments have revealed that reversible susceptibility decreases steeply with increasing IRM but that it is independent of the magnitude of TRM, ARM and memory of any types of remanence except when TRM and ARM are almost saturated. It may be concluded that the blocking mechanism of TRM and ARM is similar to the mechanism of memory of remanence but completely different from that of acquisition of IRM. It may be inferred that stable TRM, ARM and memorized remanence are carried as magnetically hard particular regions in the crystals while soft remanence is due to the more usual process of the domain wall movement.
\end{abstract}

\section{Introduction}

Thermoremanent magnetization (TRM) is known to have a very high stability. It has been shown that TRM is much more stable than isothermal remanent magnetization (IRM) against AC demagnetization (Thellier and Rimbert, 1954, 1955, Rimbert, 1956). TRM is also more stable than IRM against thermal demagnetization (Nagata, 1943, Thellier, 1941, Roquet, 1950, 1954). This high stability of TRM has provided justification of paleomagnetism: the natural remanent magnetization (NRM) of rocks can be stable enough to preserve the direction and intensity of the ancient geomagnetic field over long periods of geological time.

Several theoreis have been postulated to explain the mechanism involved in the acquisition of a stable TRM of ferromagnetic materials. In 1949 Néel proposed a theory based upon the single domain particle model. Although his theory successfully accounts for many features of TRM, the assumptions are not always applicable in rock magnetism because the particle size of the ferromagnetic minerals contained in rocks is generally larger than the critical size of single domain structure. For instance, the titanomagnetites in igneous rocks usually have the grain sizes ranging 10 to $100 \mu$, which are much larger than the theoretical estimate of the critical diameter for single domain behavior, 0.02 to $0.03 \mu$. An experiment by Syono, Akimoto and Nagata (1962) has shown that single crystals of magnetite, hematite, pyrrhotite and other synthetic ferromagnetic materials larger than several millimeters in dimension may acquire a stable TRM. Thus the theory of multidomain materials is needed to explain the origin of TRM. 
Néel (1955) developed a theory explaining how the phenomenon of magnetization blocking arises in multidomain substances during cooling from above the Curie temperature. Stacey $(1958,1963)$ proposed a theory of TRM in which the magnetic interaction energy with the applied field and the magnetostatic energy due to the self-demagnetizing field are mainly considered. In 1962 Everitt developed a similar theory. Although their theories explain some of the experimental results successfully, some results have not yet been well interpreted. In particular, the extraordinarily high stability of TRM needs more realistic explanation.

Verhoogen in 1959 postulated that the stable component of TRM originates in localized regions associated with strong internal stress around dislocations in ferromagnetic crystals. According to his consideration Néel's single domain theory may also be applicable to multidomained large particles of ferromagnetics. Although his discussion has some theoretical difficulties such as pointed by Stacey (ibid.), the basic point of his idea that TRM is not carried by the overall domain walls but that it is pinned in some localized regions should be considered carefully.

Some experimental investigations of the mechanisms involved in TRM have been made. Direct observation of the blocking process of domain walls during cooling from a temperature higher than the Curie temperature has been attempted using some transparent ferrimagnetic crystals under a high temperature microscope (Ishikawa and Syono, private communication). It has not yet been successful because the wavelength of the transmitted light goes to the infrared region far beyond the visible limit at high temperature.

The present work on the measurement of the reversible suceptibility has been carried out as an alternative access to the understanding of the remarkable distinction between TRM and IRM. It is known that the value of reversible susceptibility $\chi_{r}$ is closely related to the domain configuration of the material (Williams, 1937, Becker and Döring, 1939). Magnetization in the region of renersible susceptibility does not destroy the original domain structure of the specimen and only the fluctuation of walls and magnetic moments in the domains is observed. Therefore, it may be expected that, if the mechanism of blocking of domain orientations is different in TRM from IRM, the values of $\chi_{r}$ of a material carrying TRM will be quite different from those of the same material carrying the same amount of IRM.

Anhysteretic remanent magnetization (ARM) has also been compared with IRM in the present experiment. As shown by Néel $(1942,1943,1955)$ and Lliboutry (1951), ARM using a high AC field has some similarities with TRM. In particular its stability is closer to TRM (Rimbert, ibid., Petrova, 1959). Therefore, ARM may be used as an alternative for TRM when the artificial production of TRM is not easy because of the mechanical unstability of the crystal at high temperature.

Large crystals of hematite seem to be the most appropriate for the present study. As the intensity of saturation magnetization of hematite is small, the self-demagnetizing field in the remanence point is negligibly small compared with the coercive force even if the specimen has free poles on its ends. Therefore, we may use pieces of crystals having a bar 
or disc shape for the study of remanent magnetizaton.

Moreover, hematite has an interesting property called magnetic memory phenomenon. As reported by Haigh (1957) and Nagata, Yama-ai and Akimoto (1961), a fraction of initial remanent magnetization of hematite is recovered after the specimen is cooled to a temperature below its Morin transition (Morin, 1950) and then heated to room temperature. This recovered remanence may be called memory of remanent magnetization. The ratio of memory to the original remanence is much larger for TRM than for IRM. Stability against AC demagnetization of memory is larger than that of IRM and closer to that of stable fraction of TRM (Nagata et al, ibid.). These results imply that the mechanism of the memory phenomenon is closely connected with that of pinning of TRM. Iwata $(1963,1964)$ postulated a theoretical model for the memory phenomenon of hematite and suggested that the exchange interaction across the grain boundary, surface anisotropy energy of crystallites or the local stress in the specimen may be the origin of the pinning.

In the present experiment the susceptibility of a hematite crystal carrying a memory of remanent magnetization has also been measured in order to compare domain configuration in the state of memory with that in other types of remanent magnetization such as IRM, TRM. The mechanism of memory and its relationship with that of TRM will be discussed on the basis of this experiment.

\section{Specimens}

Two different specimens of hematite have been used in the present experiment. Descriptions of specimens together with their magnetic characteristics are given in Table 1 . Although these specimens are not really single crystals, the results may possibly be the same as in the real single crystals because the specimens are stacks of many platy single crystals the basal planes of which are parallel to one another.

Table 1. Physical Characteristics of Specimens

\begin{tabular}{c|c|c|c|c|c|c}
\hline $\begin{array}{c}\text { Specimen } \\
\text { No. }\end{array}$ & Locality & $\begin{array}{c}\text { Crystal } \\
\text { Symmetry }\end{array}$ & $\begin{array}{c}\text { Lattice } \\
\text { Const. }\end{array}$ & $\begin{array}{c}\text { Curie } \\
\text { Temp. } \\
\text { Tc }\end{array}$ & $\begin{array}{c}\text { Coercive } \\
\text { Force }\end{array}$ & Shape \\
\hline 23 & $\begin{array}{c}\text { Iron Rose } \\
\text { St. Gothard } \\
\text { Switzerland }\end{array}$ & $\begin{array}{c}\text { rhombo- } \\
\text { hedral }\end{array}$ & $\begin{array}{c}a_{r h}=5.4505 \AA \\
\alpha_{r h}=55^{\circ} 9\end{array}$ & $617^{\circ} \mathrm{C}$ & 80 oe & $\begin{array}{c}\text { Cylinder } \\
L=4.3 \mathrm{~cm} \\
D=1 \mathrm{~cm}\end{array}$ \\
\hline 25 & $\begin{array}{c}\text { Rio Marino } \\
\text { Elba }\end{array}$ & $\begin{array}{c}\text { rhombo- } \\
\text { hedral }\end{array}$ & $\begin{array}{c}a_{r h}=5.4232 \AA \\
\alpha_{r h}=55^{\circ} 14^{\prime}\end{array}$ & $6^{6} \mathrm{C}$ & 45 oe & $\begin{array}{c}\text { Disc } \\
D=3.0 \mathrm{~cm} \\
d=0.4 \mathrm{~cm}\end{array}$ \\
\hline
\end{tabular}

Specimen No. 25 is concluded to be stoichiometric $\alpha \mathrm{Fe}_{2} \mathrm{O}_{3}$ from its Curie temperature, $675^{\circ} \mathrm{C}$ and the lattice constants. This specimen exhibits the Morin transition and memory of remanent magnetization at about $-15^{\circ} \mathrm{C}$. As it appears to be very fragile and may not hold its original shape when it is heated to $800^{\circ} \mathrm{C}$, TRM has not been measured with this specimen. 
Specimen No. 23 is very solid. It can be easily heated to produce TRM or to demagnetize it thermally. However, the Curie temperature is about $60^{\circ} \mathrm{C}$ lower than that of stoichiometric hematite. This may be due to a small amount of titanium contained in this specimen. If we assume that the chemical composition is exactly on the $\mathrm{Fe}_{2} \mathrm{O}_{3}-\mathrm{FeTiO}_{3}$ solid solution line, the estimated molecular content of the $\mathrm{FeTiO}_{3}$ component is about 5 per cent. This specimen dose not exhibit the Morin trainstion at any temperature higher than the boiling temperature of liquid nitrogen $\left(-196^{\circ} \mathrm{C}\right)$.

\section{Experiment}

\subsection{Measurement of reversible susceptibility}

Reversible susceptibility has been measured by a transformer bridge designed by Fuller (1965). The bridge is first balanced carefully without the specimen and then the out-ofbalance signal in the null detector due to the insertion of the specimen into one coil is read. The linearity of the null detector reading against susceptibility has been verified experimentally as far as the susceptibility times mass of the specimen is in the order of $10^{-4} \mathrm{emu}$. The calibration of the susceptibility was carried out with $\mathrm{MnCl}_{2} \cdot 4 \mathrm{H}_{2} \mathrm{O}$ before and after each measurement in order to eliminate the effect of time drift of the balance of impedance in the bridge mainly due to the change in resistance of the coils with temperature.

The intensity of AC field of $950 \mathrm{cps}$ at the specimen during the suspectibility measurement is about $3 \propto$, which is so much smaller than the coercive force of the specimens (45$80 œ)$ that the measured susceptibility may be reversible.

\subsection{Acquisition of various types of remanent magnetization}

IRM, ARM, and TRM are produced in specimens by the usual methods. For ARM a peak intensity of AC magnetic field superposed on DC field is 900 œ. Direction of the AC field is parallel to that of DC field. The intensity of remanent magnetization is measured by an astatic magnetometer.

Fig. 1 represents curves of IRM, ARM and TRM versus applied field with specimen No. 23. Rate of acquisition of TRM is so high that TRM approaches to saturation by a field as small as 2 œ. Saturation is reached in ARM when the field is larger than about 20 œ, while IRM is far from saturation even by the field larger than $100 œ$. Intensity of saturation of remanent magnetization, $J_{r s}$, extrapolated from the data is about $2.2 \mathrm{emu} / \mathrm{gm}$ which is roughly half $(1 / 2)$ of the saturation magnetization of hematite.

\section{Results of experiment and discussion}

\subsection{Reversible susceptibility of hematite carrying IRM, ARM and TRM}

The changes in reversible susceptibility $\chi_{r}$ with increasing IRM and ARM in specimen No. 23 is shown in Fig. 2. This measurement was done with the sample in the thermally natural state prior to any heat-treatment but after demagnetization by AC magnetic field of $900 œ$ along the direction of measurment. Fig. 3 represents the change in $\chi_{r}$ of the same specimen with increasing IRM and TRM after thermal demagnetization. The initial suscep- 


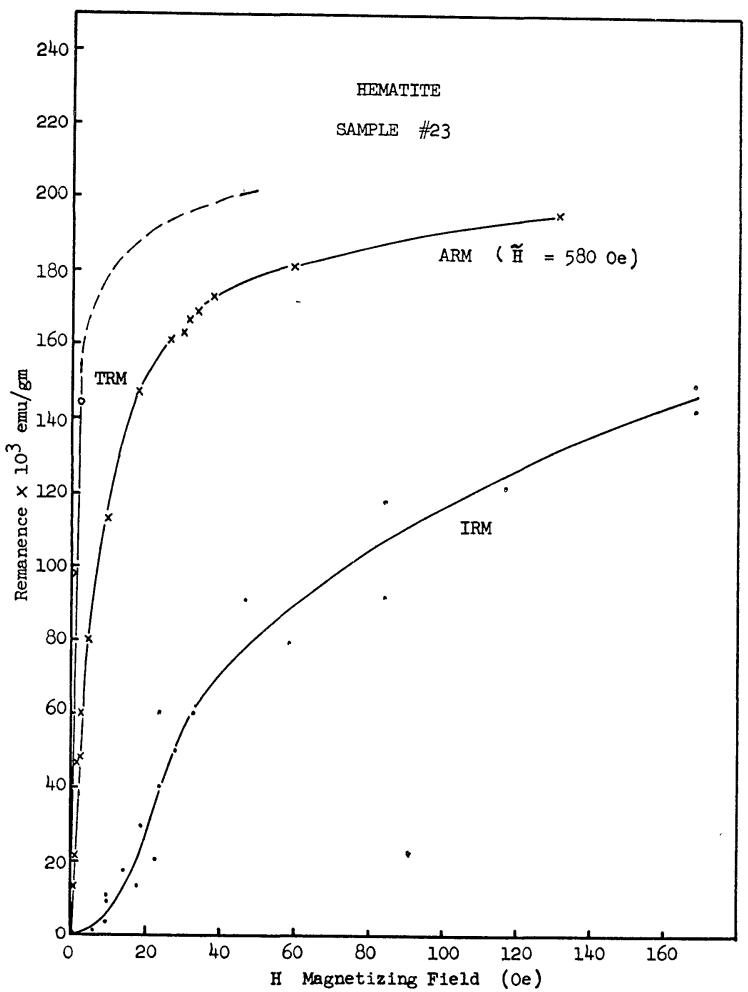

Fig. 1 Field dependence of various types of remanent magnetization (TRM, ARM, and IRM) in hematite specimen No. $23 \circ \mathrm{TRM}, \times$ ARM, · IRM

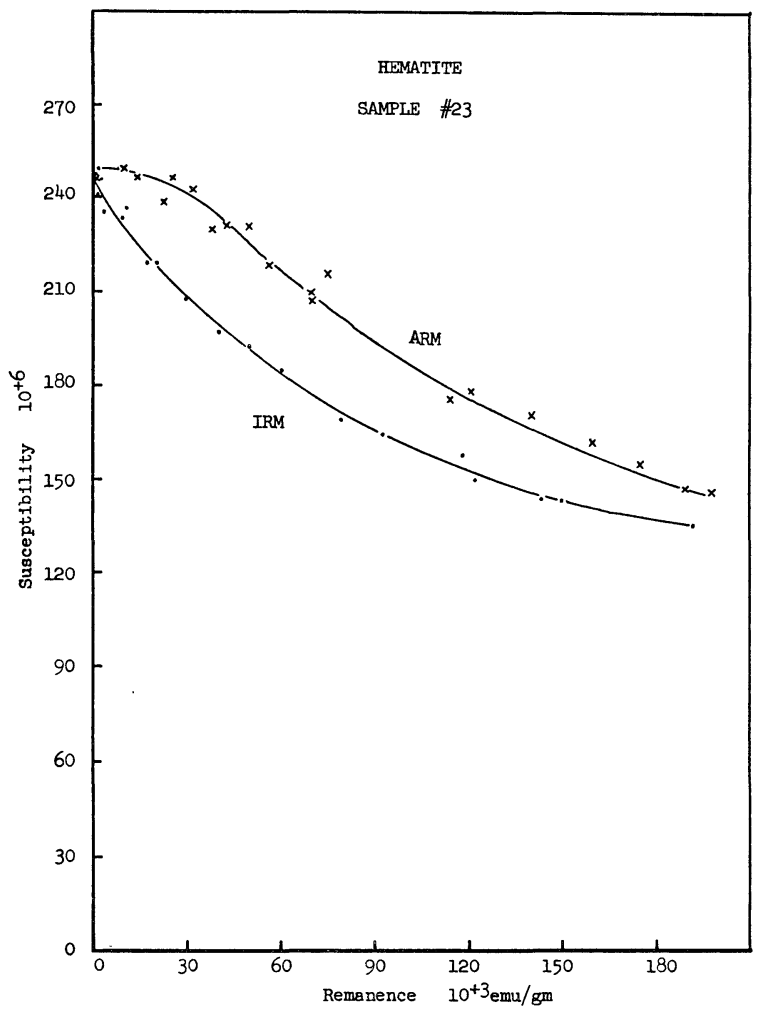

Fig. 2 Change in reversible susceptibility with IRM and ARM. Specimen No. 23, thermally natural state (demagnetized by AC magnetic field of 900 oe but prior to any heat-treatment). • IRM, $\times$ ARM 
tibility $\chi_{a}$ in the thermally demagnetized state is about 10 per cent smaller than that in the same specimen prior to any heat-treatment. It has been found that the susceptibility is very sensitive to heat-treatment, the effect of which may be one of the major problems to be examined in future. In the present experiment the curves in Fig. 3 were obtained after several repetitions of the same heat-treatmeat (slow cooling from $700^{\circ} \mathrm{C}$ ) in order to get the reproducible values of susceptibility for the subsequent TRM $-\chi_{r}$ measurment.

The $\chi_{r}-J_{r}$ curves for ARM and IRM after AC demagnetization by $900 \propto$ of the thermallytreated specimen No. 23 are also shown in Fig. 3. By the effect of the AC field the initial susceptibility $\chi_{a}$ decreases to about 90 per cent of that of the thermally demagnetized state. This result indicates that the domain configuration is rearranged so as to reduce $\chi_{a}$ by the uniaxial AC field.

Fig. 4 shows the $\chi_{r}-J_{r}$ curves for IRM and ARM in specimen No. 25. It is clearly seen in both of Figs. 3 and 4 that the susceptibility $\chi_{r}$ decreases steeply with increasing IRM but $\chi_{r}$ remains constant with increasing TRM and ARM until TRM and ARM approach saturation. This result will be discussed in the section 5.2 in this article. When the sample has an

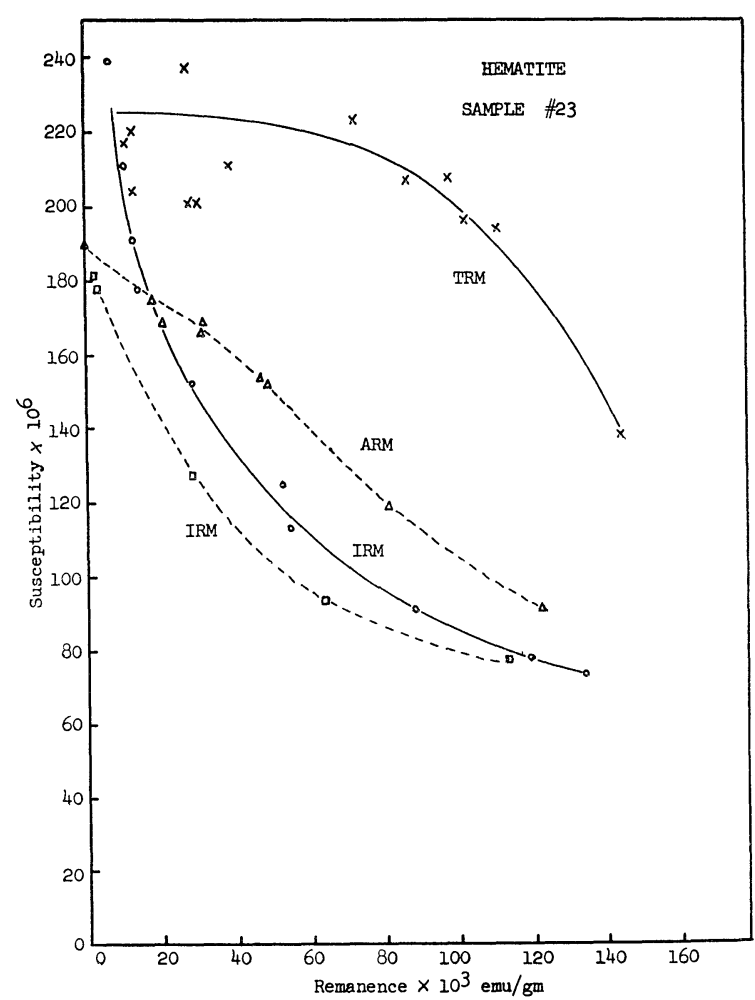

Fig. 3 Change in reversible susceptibility with various types of remanent magnetization.

Solid lines: TRM and IRM after several repetitions of slow cooling from $700^{\circ} \mathrm{C} .(\times$ TRM, $\circ$ IRM $)$ Dotted lines: after AC demagnetization by 900 oe of the thermally demagnetized state $(\triangle A R M$, $\square$ IRM) 


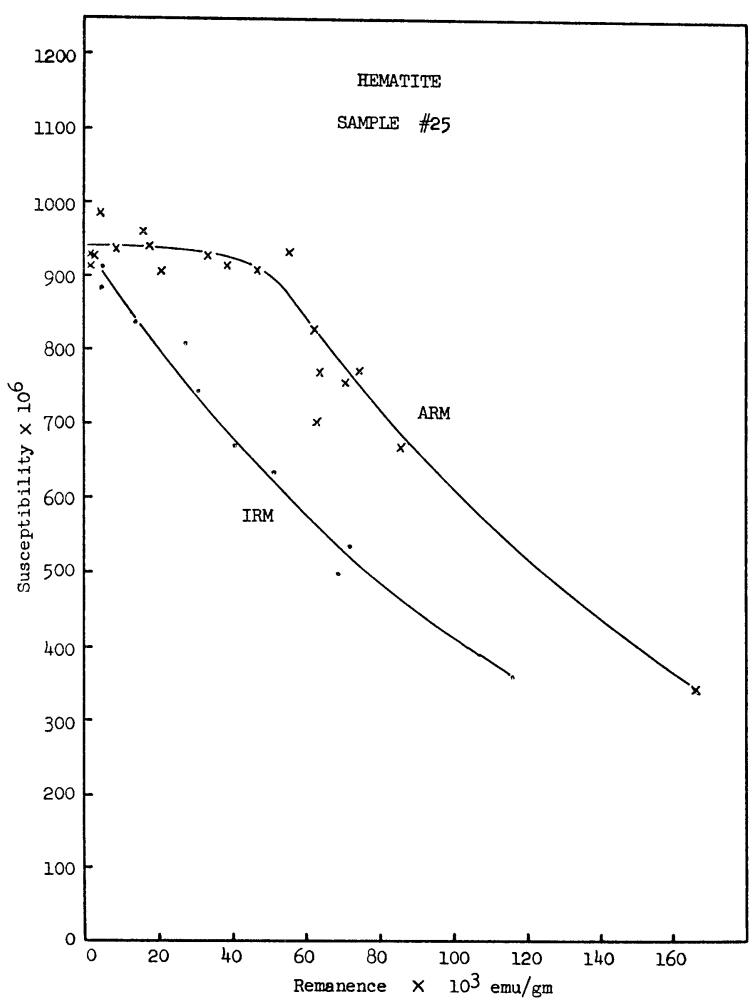

Fig. 4 Change in reversible susceptibility with ARM and IRM. Specimen No. 25 ( $\times$ ARM, · IRM)

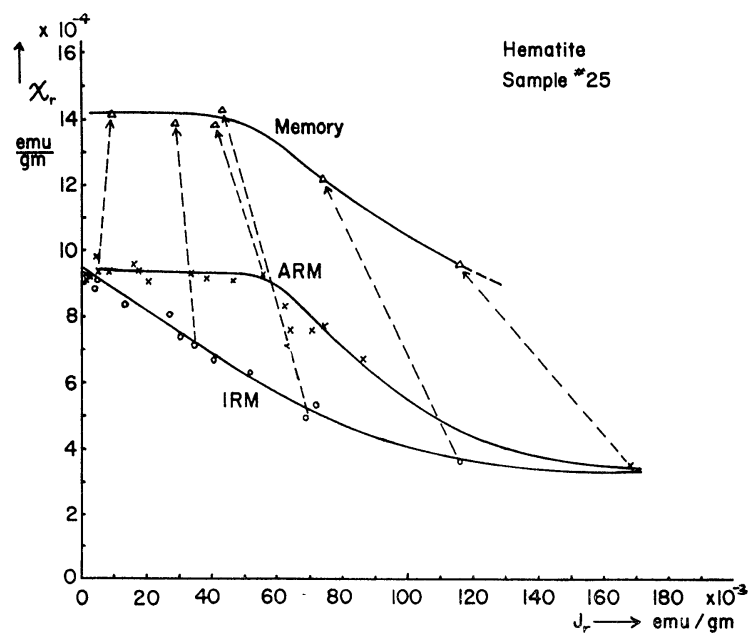

Fig. 5 Change in reversible susceptibility $\chi_{r}$ with memory of ARM and IRM. Specimen No. 25. Arrows indicate the change after one coolingheating process interposing the Morin transition. The curves for the original ARM and IRM are also shown for comparison. ( $\times$ ARM, ○IRM, $\triangle$ Memory) 
almost saturated remanence, the value of $\chi_{r}$ approaches a value $\chi_{R}$ regardless of the process of remanence, indicating that any of TRM, ARM and IRM eventually reaches the same domain configuration when the remanence is saturated.

\subsection{Reversible susceptibility of hematite carrying memory of IRM and ARM}

Fig. 5 shows the reversible susceptibility $\chi_{r}$ of specimen No. 25 carrying memory of remanence. The change in $\chi_{r}$ and $J r$ with a cooling-heating cycle interposing the Morin transition is indicated by arrows. It is remarkable that the $\chi_{r}-J r$ plots of the specimen carrying memory of remanence lie on the same curve regardless of the type of the original remanent magnetization (whether it is IRM or ARM) and that $\chi_{r}$ remains constant with increasing amount of the memory just in the same manner as with TRM or ARM.

It is also seen in Fig. 5 that the value of $\chi_{a}$ after the cooling is about 10 per cent larger than that of the $\mathrm{AC}$ demagnetized specimen. It may thus be said that the domain configuration in the specimen after the cooling process is similar to that in the thermally demagnetized specimen if we recall that $\chi_{a}$ in the thermally demagnetized state is about 10 per cent larger than that of the AC demagnetized specimen.

\section{Theoretical consideration}

\subsection{Decrease in reversible susceptibility with increasing IRM.}

The difference in the reversible susceptibility between two states of magnetization; the demagnetized state and the point of saturation remanence was discussed by Becker and Döring in their book "Ferromagnetismus" (1939). They referred to an experimental results of Dahl, Pfeffenberger and Sprung (1933) to show that the ratio of the reversible susceptibility at the remanence point $\chi_{R}$ to the initial susceptibility $\chi_{a}$ in polycrystalline nickel, iron and $40 \% \mathrm{Ni}-\mathrm{Fe}$ alloy amounts to approximately 0.3 when the stress energy is much smaller than the magnetocrystalline anisotropy energy. Becker and Döring postulated a theoretical explanation of this result on the basis of an assumption that the reversible movement of $90^{\circ}$ walls is mainly responsible for the initial and reversible susceptibility. If we take $X, Y$, $Z$ axes parallel to the easy axes of magnetization in a cubic crystal with $K>0$ such as iron, we will have 12 types of $90^{\circ}$ walls each of which is parallel to one of $(X Y),(Y Z),(Z X),\left(\bar{X} \bar{Y}^{\top}\right)$, $(\bar{Y} Z),(\bar{Z} X),(X \bar{Y}),(Y \bar{Z}),(Z \bar{X}),(\bar{X} \bar{Y}),(\bar{Y} \bar{Z}),(\bar{Z} \bar{X})$ in the demagnetized state but only three types of walls, $(X Y),(Y Z),(Z X)$ exist when the crystal has a saturation remanence in the first quadrangle.

Thus the ratio $\chi_{R} / \chi_{a}$ is given

$$
\begin{gathered}
\frac{\chi_{R}}{\chi_{a}}=\frac{4\left\{\left(\beta_{1}-\beta_{2}\right)^{2}+\left(\beta_{2}-\beta_{3}\right)^{2}+\left(\beta_{3}-\beta_{1}\right)^{2}\right\}}{\sum_{i j}^{123}\left( \pm \beta_{i} \pm \beta_{j}\right)^{2}} \\
=1-\left(\beta_{1} \beta_{2}+\beta_{2} \beta_{3}+\beta_{3} \beta_{1}\right)
\end{gathered}
$$

where $\left(\beta_{1} \beta_{2} \beta_{3}\right)$ is direction cosines of the applied field and $\sum_{i j}^{123}\left( \pm \beta_{i} \pm \beta_{j}\right)^{2}$ indicates the sum of 12 terms ; $\left(\beta_{1}-\beta_{2}\right)^{2},\left(\beta_{1}-\beta_{2}\right)^{2},\left(-\beta_{1}+\beta_{2}\right)^{2}$, etc., which is equal to 8 . If the sample is an assembly of randomly oriented crystallites, 


$$
\frac{\chi_{R}}{\chi_{a}}=1-\overline{\left(\beta_{1} \beta_{2}+\beta_{2} \beta_{3}+\beta_{3} \beta_{1}\right)}=1-\frac{2}{\pi}=0.346
$$

In case of nickel $(K<0), \chi_{R} / \chi_{a}=0.328$.

This ratio appears to be quite consistent with the present result on hematite in which $\chi_{R} / \chi_{a}$ is about 0.3 to 0.5 although the domain structure in hematite is not well-known.

In the actual specimens the magnetic hardness of domains has a wide distribution. Thus soft domains are first magnetized and hold IRM. Then the number of domains turned to the field direction linearly increases with increasing IRM. It may, therefore, be expected that the susceptibility decreases proportionally to the increase in the intensity of IRM. The results in Figs. 3, 4 and 5 show that this is roughly true. In both specimens this linear decrease in $\chi_{r}$ with IRM holds until the IRM reaches about $2 / 3$ of the saturation remanence. This may imply that about $2 / 3$ of the total walls are sufficiently soft to contribute to reversible susceptibility and acquisition of IRM.

\subsection{Independence of reversible susceptibility to stable remanence and memory.... Origin of TRM and memory}

The experimental result that the reversible susceptibility is independent of the magnitude of TRM and ARM indicates that TRM and ARM are not carried by the usual soft domains which are responsible for IRM and reversible susceptibility. The regions carrying TRM and ARM seem to be magnetically much harder, their walls being blocked tightly with certain structures at certain position. Since this independent nature of stable remanence holds until the amount of remanence reaches about $1 / 3$ of that of the saturation remanence, it may be said that about $1 / 3$ of the total domains are hard in this specimen. After the amount of remanence exceeds the limit, the susceptibility decreases linearly with increasing amount of remanence just as in the initial part of the $\chi_{r}$ versus IRM curve. When saturation is reached, the susceptibility seems to approach the same value $\chi_{R}$ regardless the type of the remanence. Therefore, it is rather unlikely that the stability (hardness) of TRM is induced in the overall walls by a particular mechanism during cooling in a weak field, e.g., the locally induced anisotropy due to the magnetic field cooling effect. The experimental results are more favorable to the hypothesis that a number of particularly hard regions which may carry stable remanence exist in the crystal. When a strong saturating field is applied isothermally, both soft and hard portions are magnetized, then they hold a saturation IRM. The same state of remanence is reached if a reasonably large DC field is applied through TRM or ARM process. In this case the hard fractions are magnetized by a usual mechanism of TRM or ARM but even the soft fractions are magnetized by the IRM mechanism. Thus the magnetic field required to reach saturation of remanence may be much smaller than that required with the pure IRM process, which is clearly seen in the $J r-H$ curves such as Fig. 1.

Measurement of susceptibility of stoichiometric hematite after a cooling-heating treatment through the Morin transition seems to reveal a remarkable similarity of memorized remanence with TRM and ARM. The result indicates that the memory is also carried by the particularly hard regions which are not responsible for the reversible susceptibility. 
When the specimen is cooled through the Morin transition, the remanence carried by the soft fraction is completely lost, but the hard regions hold the latent memory of remanence below the transiton. Then the hard fractions of remanence recover entirely after the specimen is warmed to temperatures above the transition.

For this phenomenon of magnetic memory Iwata (ibid.) postulated models as stated in the introduction of this article. His first consideration involving the effect of the grain boundary or the surface anisotropy of crystallites does not seem to be valid with this single crystal.

Recently Tasaki and Iida (1964) has shown that the energy of magnetoelastic coupling in the basal plane of hematite has a great importance in the memory phenomena. Blackman and his co-worker (private communication) have observed change in the Bitter pattern of hematite between $20^{\circ} \mathrm{C}$ and $-30^{\circ} \mathrm{C}$ using the $\mathrm{Fe}_{3} \mathrm{O}_{4}$ colloids suspended in alcohol. Their movie film clearly indicates that the once-memorized walls reappear at the same positions when the specimen is warmed through the transition during the subsequent cooling-heating cycles.

By recent investigation of the memory phenomena in magnetite, a model has been proposed to explain the common origin of memorized remanence and stable fraction of remanent magnetization (Fuller and Kobayashi, 1965). In their model it has been postulated that the internal stress field around dislocations pins a $90^{\circ}$ wall under a certain favorable condition and that direction of a magnetic domain surrounded by the $90^{\circ}$ wall is uniquely decided by the spin configuration in the wall. As the stress is uniaxial, the pinned domains are also uniaxially anisotropic. Magnetization of the region may be only due to the $180^{\circ}$ rotation of a set of domains around the pinned wall. Thus this region may be magnetically so hard at ordinary tempearture that it may be responsible for the TRM (or ARM) and memory.

\section{Acknowledgments}

We are grateful to Professor T. Nagata for his continuous encouragement through this study. We are also grateful to Dr. M. Fuller for the design of the susceptibility bridge and to Dr. Y. Ishikawa for his invaluable suggestion. We acknowledge Dr. E. R. Eller, Carnegie Museum, Pittsburgh for his kindness in putting his specimens at our disposal.

This research has been supported by grants from the U.S. National Science Foundation, research grants GP-423, GP-4058 and GF-75.

\section{References}

Becker, R. and W. Döring : "Ferromagnetismus" 1939, Springer, Berlin.

Blackman, M. and B. Gustard, Domains in haematite, Nature, 193, 360-361, 1962.

Dahl, O., J. Pfaffenberger and H. Spring, Elektr. Nacher. Techn. Bd. 10, 317- , 1933 quoted in Becker and Döring's "Ferromagnetismus" (1939) p. 161.

Everitt, C. W. F., Thermoremanent magnetization (III), Theory of multidomain grains, Phil. Mag., 7, 599-616, 1962.

Fuller, M. D., AC bridge methods for the measurement of susceptibility anisotropy in " Methods and Techniques in Palaeomagnetism", ed. by D. W. Collinson and K. M. Creer, Elsevier Co. (in Press).

Fuller, M. D. and Kazuo Kobayashi, Dislocation model of memory and stable fraction of remanent magnetization in multidomain ferromagnetics, to be published 
Haigh, G., Observations on the magnetic transition in hematite at $-15^{\circ} \mathrm{C}$, Phil. Mag. 2, 878-890, 1957.

Iwata, T., Mechanism of the memory phenomenon in $\alpha \mathrm{Fe}_{2} \mathrm{O}_{3}$, J. Phys. Soc. Japan, 18, 1100, 1963.

Iwata, T., A generalized model for the momory phenomenon of $\alpha \mathrm{Fe}_{2} \mathrm{O}_{3}$, J. Phys. Soc. Japan, 19, 583-584, 1964.

Lliboutry, L., L'aimantation des aciers dans les champs magnétiques faibles: effets du temps, des tensions, des chocs, des champs magnétiques transversaux, Ann. physique 6, 731-829, 1951

Morin, F. J., Magnetic susceptibility of $\alpha \mathrm{Fe}_{2} \mathrm{O}_{3}$ and $\alpha \mathrm{Fe}_{2} \mathrm{O}_{3}$ with added titanium, Phys. Rev., 78, 819-820, 1950.

Nagata, T., “Rock Magnetism ”, Maruzen Co., Tokyo, 1st ed. 225 pp., 1953, rev. ed. 350 pp., 1961.

Nagata, T., Natural remanent magnetization of volcanic rocks and its relation to geophysical phenomena, Bull. Earthq. Res. Inst. 21, 1-196, 1943

Nagata, T., M. Yama-ai and S. Akimoto, Memory of initial remanent magnetization and number of repeating of heat treatments in low-temperature behavior of hematite, Nature 190, 620-621, 1961.

Néel, L., Theorie de l'effet du champ demagnetisant sur l'aimantation anhysteretique, Cahiers phys., 17, 47-50, 1943.

Néel, L., Theorie du trainage magnetique des ferromagnetiques en grains fins avec applications aux terres cuites, Ann. geophys. 5, 99-136, 1949.

Néel, L., Some physical aspects of rock magnetism, Adv. Phys. 4, 191-243, 1955.

Petrova, G. N., Magnetic stability of rocks, Izv. Akad. Nauk. USSR, ser. Geophys., 52-61, 1956.

Rimbert, F., Contribution a l'etude de l'action de champs alternatifs sur les aimantations remanentes des roches, Applications geophysiques, Rev. Inst. franc. petrole et Ann. combustibles, liquides, 14, 17-54, and 123-155, 1959.

Roquet, J., Sur la remanence des oxydes de fer et leur interet en geomagnetisme, Ann. geophys., 10, 226-247, 1954.

Roquet, J., Sur les aimantations thermoremanente isotherme du sesquioxyde de fer et de la magnetite, J. Geomag. Geoelec. 6, 200-205, 1954.

Siratori, K., A. Tasaki and S. Iida, On the mechanism of reversible appearance of residual magnetization at low temperature transition in $\alpha \mathrm{Fe}_{2} \mathrm{O}_{3}$, J. Phys. Soc. Japan, 15, 2357-2358, 1960.

Stacey, F. D., Thermo-remanent magnetization (TRM) of multidomain grains in igneous rocks, Phil. Mag. 3, 1391-1401, 1958.

Stacey, F. D., Physical theory of rock magnetism, Adv. Phys., 12, 46-133, 1963.

Syono, Y., S. Akimoto and T. Nagata, Remanent magnetization of ferromagnetic single crystals, J. Geomag. Geoelec., 14, 113-124 (1962).

Tasaki, A. and S. Iida, Memory phenomenon of $\alpha \mathrm{Fe}_{2} \mathrm{O}_{3}$ powder made from single crystal, J. Phys. Soc. Japan, 19, 1091, 1964.

Thellier, E., Sur les proprietes de l'aimantation thermoremanente des terres cuites, C.R. Acad. Sci., Paris, 213, 1019-1022, 1941.

Thellier, E. and F. Rimbert, Sur l'analyse d'aimantations fossiles par action de champs magnetiques alternatifs. C.R. Acad. Sci., Paris, 239, 1399-1401, 1954.

Thellier, E. and F. Rimbert, Sur l'utilisation en paleomagnetisme de la desaimantation par champs alternatif C.R. Acad. Sci., Paris, 240, 1404-1406, 1955.

Verhoogen, J., The origin of thermo-remanent magnetization, J. Geophys. Res., 64, 2441-2449, 1959.

Williams, H. J., Magnetic properties of single crystals of Si-Fe, Phys. Rev., 52, 747-751, 1937. 